International Journal of Applied Mathematics

Volume 34 No. $4 \quad 2021,633-646$

ISSN: 1311-1728 (printed version); ISSN: 1314-8060 (on-line version)

doi: http://dx.doi.org/10.12732/ijam.v34i4.3

\title{
SOME VARIANTS OF THE CONTRACTION MAPPING PRINCIPLE FOR COMPARABLE ELEMENTS
}

\author{
El Khannoussi Mohammed Said ${ }^{1}$, Abderrahim Zertiti ${ }^{2}$ \\ ${ }^{1,2}$ University Abdelmalek Essaadi \\ Faculty of Sciences, Department of Mathematics \\ BP 2121, Tetouan, MOROCCO
}

\begin{abstract}
In this paper, by using the partial ordering method we study the existence and uniqueness of fixed points for operator equations. The convergence of the iterative sequences to fixed points is satisfied. The presented theorems improve and generalize some important results in the literature.
\end{abstract}

AMS Subject Classification: 47H10, 55M20, 12H20

Key Words: Banach contraction principle; operator equations; spectral radius; successive approximations; differential equations

\section{Introduction}

The well-known Banach contraction principle states that if $(X, d)$ is a complete metric space and $f:(X, d) \rightarrow(X, d)$ is a contraction i.e.,

$$
d(f(x), f(y)) \leq \alpha d(x, y),
$$

for all $x, y \in X$, where $0 \leq \alpha<1$, then $f$ has a unique fixed point. This result has been extensively used in solving various problems in different research fields. As well as, there are a lot of extensions of this famous fixed point theorem, for instance, in [9], Krasnosel'skii and Zabreiko presented the following variant of this result when one requires particular estimates only for the deferences of the values of the operator on comparable elements.

Received: January 22, 2021

(c) 2021 Academic Publications

${ }^{\S}$ Correspondence author 
Theorem A. Let $(E, P)$ be an ordered Banach space with a normal reproducing cone $P$ and let $A: E \rightarrow E$ be an operator. If there exists a positive linear boundary operator $L: E \rightarrow E$ with spectral radius $\sigma(L)<1$ satisfying

$$
-L(x-y) \leq A(x)-A(y) \leq L(x-y), \quad x, y \in E, \quad x \geq y,
$$

then $A$ has a unique fixed point $x *$ in $E$ and for any $x_{0} \in E$ if $x_{n}=A x_{n-1}(n=$ $1,2,3, \ldots$.$) , then x_{n} \rightarrow x *$ as $n \rightarrow \infty$.

In the present paper we extend this important result. Moreover, we weaken the hypothesis $\sigma(L)<1$ by suggesting new conditions: $L$ have the property of $u_{0}$ - boundedness above and satisfies the inequality $L u_{0} \leq \lambda u_{0}$ for some $\lambda \in[0,1)$. Furthermore, if the operator $A$ leaves invariant the cone $P$ we present another result in the case where the cone $P$ is not necessarily generating. The proof will be based on the partial ordering method (see [5, 4]. Finally and in order to demonstrate the importance of our abstract results application to the existence of solutions to nonlinear boundary value problem is presented.

\section{Main results}

Let $\left(E,\|\cdot\|_{E}\right)$ be a real Banach space and $P$ be a nonempty closed convex set in $E$.

$P$ is called a cone if it satisfies the following two conditions:

(i) : $x \in P, \lambda \geq 0 \Longrightarrow \lambda x \in P$,

(ii) : $x \in P,-x \in P \Longrightarrow x=\theta$, where $\theta$ denotes the zero element in $E$.

A cone $P$ is said to be generating (or reproducing) if $E=P-P$, i.e., every element $x \in E$ can be represented in the form $x=u-v$ where $u, v \in P$.

The cone $P$ defines a linear ordering in $E$ by

$$
x \leq y \quad \text { iff } \quad y-x \in P .
$$

The cone $P$ is said to be normal if there exists a constant $N>0$ such that

$$
\theta \leq x \leq y \Longrightarrow\|x\| \leq N\|y\|, \quad x, y \in P .
$$

For every $L: E \rightarrow E$ a bounded linear operator, define $\sigma(L)$, the spectral radius of $L$ by

$$
\sigma(L)=\lim _{n \rightarrow+\infty}\left\|L^{n}\right\|^{\frac{1}{n}} .
$$


The linear operator $L$ is called positive if it transforms the cone $P$ into itself. It is not hard to see that it follows from $x \leq y$ for arbitrary elements $x, y \in E$ that $L x \leq L y$.

We denote by $u_{0}$ some fixed non-zero element of $P$. Following Krasnosel'skii [10], we call the linear positive operator $L, u_{0}$-bounded above if for every nonzero element $x \in P$ a natural number $m$ and a positive number $\beta$ can be found such that

$$
L^{m} x \leq \beta u_{0}
$$

After these preparations we are ready to prove this variant of the well-known Weissinger's Theorem (see [13]) for comparable element.

Theorem 1. Let $(E, P)$ be an ordered Banach space with normal generating cone $P$ and $A: E \rightarrow E$ be an operator. Suppose that there exist positive linear operators $L_{n}: E \rightarrow E(n=1,2, \ldots)$ such that

$$
-L_{n}(x-y) \leq A^{n}(x)-A^{n}(y) \leq L_{n}(x-y), \quad x, y \in E, \quad x \geq y .
$$

If $\sum_{n=0}^{\infty} L_{n}(v)$ exists for all $v \in P$, then $A$ has a unique fixed point $x^{*}$ in $E$ and for any $x_{0} \in E$, if $x_{n}=A x_{n-1}(n=1,2,3, \ldots)$, then $x_{n} \rightarrow x^{*}$ as $n \rightarrow \infty$. Furthermore, for some element $u \in P$ dependent of $x_{0}$ we have the following estimate of convergence rate

$$
\left\|x_{n}-x^{*}\right\| \leq(2 K+1)\left\|\sum_{i=n}^{\infty} L_{i}(u)\right\|,
$$

where $K$ is the normal constant of $P$.

Proof. Since $P$ is generating then for an arbitrary $x \in E$ elements $y, z \in P$ can be found such that $x=y-z$, which implies that

$$
-(y+z) \leq x \leq y+z
$$

This means that for any element $x \in E$ there exists an element $u \in P$ satisfying $-u \leq x \leq u$. Fix an $x_{0} \in E$ and set $x_{n}=A^{n} x_{0}, n=1,2, \ldots$, then there exists an element $u \in P$ such that

$$
-u \leq x_{1}-x_{0} \leq u
$$

hence

$$
x_{1} \geq \frac{1}{2}\left(x_{1}+x_{0}-u\right), \quad x_{0} \geq \frac{1}{2}\left(x_{1}+x_{0}-u\right) .
$$


By using inequalities (1) we get for every $n=1,2, \ldots$ :

$$
-L_{n}\left(\frac{x_{1}-x_{0}+u}{2}\right) \leq x_{n+1}-A^{n}\left(\frac{x_{1}+x_{0}-u}{2}\right) \leq L_{n}\left(\frac{x_{1}-x_{0}+u}{2}\right)
$$

and

$$
-L_{n}\left(\frac{x_{0}-x_{1}+u}{2}\right) \leq x_{n}-A^{n}\left(\frac{x_{1}+x_{0}-u}{2}\right) \leq L_{n}\left(\frac{x_{0}-x_{1}+u}{2}\right) .
$$

By subtracting (3) for (2), then we have

$$
-L_{n} u \leq x_{n+1}-x_{n} \leq L_{n} u .
$$

As a consequence of the last inequality we obtain for $n>m \geq 1$

$$
-\sum_{i=m}^{n-1} L_{i} u \leq x_{n}-x_{m} \leq \sum_{i=m}^{n-1} L_{i} u
$$

from which it follows that

$$
0 \leq x_{n}-x_{m}+\sum_{i=m}^{n-1} L_{i} u \leq 2 \sum_{i=m}^{n-1} L_{i} u .
$$

Using the normality of the cone there is a $K>0$ such that

$$
\left\|x_{n}-x_{m}+\sum_{i=m}^{n-1} L_{i} u\right\| \leq 2 K\left\|\sum_{i=m}^{n-1} L_{i} u\right\| .
$$

This implies that

$$
\left\|x_{n}-x_{m}\right\| \leq\left\|x_{n}-x_{m}+\sum_{i=m}^{n-1} L_{i} u\right\|+\left\|\sum_{i=m}^{n-1} L_{i} u\right\| \leq(2 K+1)\left\|\sum_{i=m}^{n-1} L_{i} u\right\| .
$$

On the other hand, it is clear that $\sum_{i=0}^{n} L^{i} u(n=1,2, \ldots)$ is a Cauchy sequence, and so $\left(x_{n}\right)_{n=0}^{\infty}$ is a Cauchy sequence. Since $E$ is a Banach space the sequence converges, i.e., there exists a $x^{*} \in E$ such that $x_{n} \rightarrow x^{*}$ as $n \rightarrow \infty$. In order that $x^{*}$ be a fixed point of $A$ it suffices to prove that $A$ is continuous. Indeed, let $x, y \in E$, since $P$ is generating, there exist (see [4, Lemma 1.4.2]) $\tau>0$ and element $z, w \in P$ such that $x-y=z-w$ with $\|z\| \leq \tau\|x-y\|,\|w\| \leq \tau\|x-y\|$. By using the same arguments as above, then it follows from

$$
-(z+w) \leq x-y \leq z+w
$$


that

$$
-L_{1}(z+w) \leq A(x)-A(y) \leq L_{1}(z+w),
$$

which in turn implies that

$$
\|A(x)-A(y)\| \leq(2 K+1)\left\|L_{1}(z+w)\right\| .
$$

By using the fact that a linear operator which maps a reproducing cone into a normal cone must be continuous (see [9]) we get

$$
\|A(x)-A(y)\| \leq(2 K+1)\left\|L_{1}\right\|\|z+w\| .
$$

This yields

$$
\|A(x)-A(y)\| \leq(2 K+1)(2 \tau)\left\|L_{1}\right\|\|x-y\|
$$

that is, the operator $A$ is uniformly continuous.

From the above argument it follows that $\left(A^{n}\left(x_{0}\right)\right)_{n=1}^{\infty}$ converges to the unique fixed point independently of the choice of $x_{0} \in E$. In fact, let $y_{0} \in E$, and take an element $v \in P$ such that $-v \leq y_{0}-x^{*} \leq v$. By the same argument used above we find

$$
-L_{n} v \leq y_{n}-x^{*} \leq L_{n} v, \quad y_{n}=A^{n} y_{0}, \quad n=1,2, \ldots
$$

By using the normality of the cone and the equality $\lim _{n \rightarrow \infty} L_{n}(v)=0$ we derive that $\lim _{n \rightarrow \infty} y_{n}=x^{*}$.

Similarly, we can prove that $x^{*}$ is the unique fixed point of $A$. Indeed, suppose that $\bar{x}$ is another fixed point of $A$ and consider the sequence $\bar{x}_{n}=$ $A^{n} \bar{x}=\bar{x}$, we obtain $\bar{x}=x^{*}$.

Finally, by letting $n \rightarrow \infty$ in (4) we get

$$
\left\|x^{*}-x_{n}\right\| \leq(2 K+1)\left\|\sum_{i=n}^{\infty} L_{i} u\right\| .
$$

This completes the proof of the theorem.

The following lemma will be used latter.

Lemma 2. Let $(E, P)$ be an ordered Banach space with normal cone $P$ and $L: E \rightarrow E$ be a $u_{0}$-bounded above operator such that for some $\lambda \in$ $[0,1), \quad L u_{0} \leq \lambda u_{0}$. Then $I-L$ is invertible in the cone $P$ and we have

$$
(I-L)^{-1}=\sum_{n=0}^{\infty} L^{n}=\lim _{n \rightarrow \infty}\left(I+L+L^{2}+\ldots+L^{n}\right) .
$$


Proof. Choose an element $x \in P$, then from the $u_{0}$-boundedness above of $L$ a natural number $m$ and a positive number $\beta$ can be found such that

$$
L^{m} x \leq \beta u_{0}
$$

Hence

$$
L^{m+1} x \leq \beta L u_{0} \leq \beta \lambda u_{0},
$$

from which it follows that

$$
L^{m+n} x \leq \beta \lambda^{n} u_{0}, \quad n=0,1,2, \ldots
$$

Since $\left(\sum_{i=0}^{n} \beta \lambda^{i} u_{0}\right)_{n \in \mathbb{N}}$ is a Cauchy sequence, there is an $N$ such that for all $p>q \geq N,\left\|\beta \lambda^{q+1} u_{0}+\beta \lambda^{q+2} u_{0}+\ldots+\beta \lambda^{p} u_{0}\right\|<\varepsilon$. Therefore and by setting $S_{n}(x)=\sum_{i=0}^{n} L^{i} x$, we obtain

$$
\begin{aligned}
0 & \leq S_{p+m}(x)-S_{q+m}(x) \\
& =L^{q+m+1}(x)+L^{q+m+2}(x)+\ldots+L^{p+m}(x) \\
& \leq \beta \lambda^{q+1} u_{0}+\beta \lambda^{q+2} u_{0}+\ldots+\beta \lambda^{p} u_{0} .
\end{aligned}
$$

Using the normality of the cone there is a positive constant $K$ such that

$$
\left\|S_{p+m}(x)-S_{q+m}(x)\right\| \leq K\left\|\beta \lambda^{q+1} u_{0}+\beta \lambda^{q+2} u_{0}+\ldots+\beta \lambda^{p} u_{0}\right\|<K \varepsilon .
$$

This implies that $S_{n}(x)(n=0,1,2 \ldots)$ is a Cauchy sequence in $E$ then it converges and we can note that for all $x \in P, S(x)=\lim _{n \rightarrow \infty} S_{n}(x)$.

We now need to show that $S(I-L)=(I-L) S=I$ in $P$.

Let $x$ be an element of the cone $P$, we have

$$
\begin{aligned}
S(I-L)(x) & =S(x)-S L(x) \\
& =\lim _{n \rightarrow \infty} S_{n}(x)-\lim _{n \rightarrow \infty} S_{n}(L x) \\
& =\lim _{n \rightarrow \infty}\left(S_{n}(x)-S_{n}(L x)\right) \\
& =\lim _{n \rightarrow \infty}\left(x-L^{n+1} x\right) \\
& =x .
\end{aligned}
$$

Here we use the fact that $\lim _{n \rightarrow \infty} L^{n}(x)=0$ which can be obtained from the equality $L^{n}(x)=S_{n}(x)-S_{n-1}(x)$.

A similar argument shows that $(I-L) S=I$ in $P$. This completes the proof of the lemma. 
As a consequence of Theorem 1 and Lemma 2 we present the following statement.

Theorem 3. Let $(E, P)$ be an ordered Banach space with normal generating cone $P$ and $A: E \rightarrow E$ be an operator. Suppose that there exists an $u_{0}$-bounded above linear operator $L: E \rightarrow E$ such that

$$
-L(x-y) \leq A(x)-A(y) \leq L(x-y), \quad x, y \in E, \quad x \geq y
$$

If $L u_{0} \leq \lambda u_{0}$ for some $\lambda \in[0,1)$, then $A$ has a unique fixed point $x^{*}$ in $E$ and for any $x_{0} \in E$, if $x_{n}=A x_{n-1}(n=1,2,3, \ldots)$, then $x_{n} \rightarrow x^{*}$ as $n \rightarrow \infty$. Furthermore, for some element $u \in P$ dependent of $x_{0}$ we have the following estimate of convergence rate

$$
\left\|x_{n}-x^{*}\right\| \leq(2 K+1)\left\|\sum_{i=n}^{\infty} L^{i}(u)\right\|,
$$

where $K$ is the normal constant of $P$.

Proof. We are going to see that all conditions of Theorem 1 are verified. In fact, Let $x, y \in E, \quad x \geq y$, then we get

$$
-L(x-y) \leq A(x)-A(y) \leq L(x-y),
$$

Making the substitutions $u=L(x-y) \in P, \quad X=A(x), Y=A(y)$, then

$$
-u \leq X-Y \leq u \text {. }
$$

Thus, by the same arguments used in the proof of Theorem 1 we get

$$
-L u \leq A X-A Y \leq L u
$$

This yields that

$$
-L^{2}(x-y) \leq A^{2} x-A^{2} y \leq L^{2}(x-y)
$$

Repeating this argument $n-1$ time we obtain

$$
-L^{n}(x-y) \leq A^{n} x-A^{n} y \leq L^{n}(x-y) .
$$

By virtue of Lemma $2, \sum_{n=0}^{\infty} L^{n} v$ exists for any $v \in P$, hence the conclusion of the theorem can be deduced from Theorem 1 . 
Remark 4. If we suppose in the last theorem that $P$ is a solid cone and that $u_{0}$ belong to the interior of the cone $P$ then it follows from [4, Theorem 1.4.1] that for any $f \in P^{*} \backslash\{0\}$ (where $P^{*}$ is the dual cone of $P$ ) we have $f\left(u_{0}\right)>0$, that is, $u_{0}$ is a quasi-interior element of the cone in the sense of Krasnosel'skii [14]. Further, fix an element $x \in P$, then for a sufficiently large $\beta>0$ the element $u_{0}-\frac{1}{\beta} L x$ will belongs to the cone, which implies that $L$ is $u_{0^{-}}$ bounded above. Consequently, it follows from the existence of some $\lambda \in[0,1)$ satisfying $L u_{0} \leq \lambda u_{0}$ that $\sigma(L)<1$ (see [14, Theorem 2]).

Remark 5. If $A$ satisfies the conditions of anyone of the above theorems then the equation $x=A x+y$ has a unique solution in $E$ for each $y \in E$.

Remark 6. Suppose that $L: E \rightarrow E$ is a positive linear boundary operator $L: E \rightarrow E$ with spectral radius $\sigma(L)<1$, then it is well known that $I-L$ is invertible with

$$
(I-L)^{-1}=\sum_{n=0}^{\infty} L^{n}=\lim _{n \rightarrow \infty}\left(I+L+L^{2}+\ldots+L^{n}\right) .
$$

This implies that we can get Theorem A by an application of Theorem 1.

Now, we are going to see that if the cone $P$ is not necessarily generating, then similar results can be obtained in the case of the positiveness of the operator $A(A(P) \subset P)$.

Theorem 7. Let $(E, P)$ be an ordered Banach space with normal cone $P$ and $A: E \rightarrow E$ be a positive continuous operator. Suppose that there exist positive linear operators $L_{n}: E \rightarrow E(n=1,2, \ldots)$ such that

$$
-L_{n}(x-y) \leq A^{n}(x)-A^{n}(y) \leq L_{n}(x-y), \quad x, y \in E, \quad x \geq y .
$$

If $\sum_{i=0}^{\infty} L_{i}(v)$ exists for all $v \in P$, then $A$ has a unique fixed point $x^{*}$ in $P$ and for any $x_{0} \in P$, if $x_{n}=A x_{n-1}(n=1,2,3, \ldots$.$) , then x_{n} \rightarrow x^{*}$ as $n \rightarrow \infty$. Furthermore, we have the following estimate of convergence rate

$$
\left\|A^{n}(0)-x^{*}\right\| \leq(2 K+1)\left\|\sum_{i=n}^{\infty} L_{i}(A(0))\right\| .
$$

Proof. By observing that $A(0) \geq 0$ and by setting $x_{n}=A^{n}(0)$ we have for $n=1,2, \ldots$

$$
-L_{n}\left(x_{1}\right) \leq x_{n+1}-x_{n} \leq L_{n}\left(x_{1}\right), \quad x, y \in E, \quad x \geq y .
$$


Now the the proof can be completed by the same arguments used in Theorem 1.

Similarly, we have the following statement.

Theorem 8. Let $(E, P)$ be an ordered Banach space with normal cone $P$ and $A: E \rightarrow E$ be a positive continuous operator. Suppose that there exists an $u_{0}$-bounded above linear operator $L: E \rightarrow E$ such that

$$
-L(x-y) \leq A(x)-A(y) \leq L(x-y), \quad x, y \in E, \quad x \geq y .
$$

If $L u_{0} \leq \lambda u_{0}$ for some $\lambda \in[0,1)$, then $A$ has a unique fixed point $x^{*}$ in $P$ and for any $x_{0} \in P$, if $x_{n}=A x_{n-1}(n=1,2,3, \ldots)$, then $x_{n} \rightarrow x^{*}$ as $n \rightarrow \infty$. Furthermore, we have the following estimate of convergence rate

$$
\left\|A^{n}(0)-x^{*}\right\| \leq(2 K+1)\left\|\sum_{i=n}^{\infty} L^{i}(A(0))\right\| .
$$

It should be remarked above that Theorem A remains valid if we replace condition (5) by the assumption that

$$
-L_{1}(x-y) \leq A(x)-A(y) \leq L_{2}(x-y), \quad x, y \in E, \quad x \geq y,
$$

where $L_{1}$ and $L_{2}$ are positive linear operators with $\sigma\left(L_{1}+L_{2}\right)<1$. Here, observe that even if $A$ is a linear operator the latter condition cannot be replaced by the inequalities $\sigma\left(L_{1}\right)<1$ and $\sigma\left(L_{2}\right)<1$. In the following statement we study this problem by suggesting new conditions concerning every operators $L_{1}$ and $L_{2}$.

Theorem 9. Let $(E, P)$ be an ordered Banach space with normal generating cone $P$ and $A: E \rightarrow E$ be an operator. Suppose that there exist linear operators $L_{1}, L_{2}: E \rightarrow E$ such that

$$
-L_{1}(x-y) \leq A(x)-A(y) \leq L_{2}(x-y), \quad x, y \in E, \quad x \geq y .
$$

Assume that $L_{1}+L_{2}$ is $u_{0}$-bounded above, then if $L_{1} u_{0} \leq \lambda_{1} u_{0}$ and $L_{2} u_{0} \leq \lambda_{2} u_{0}$ for some $\lambda_{1}, \lambda_{2} \geq 0$ satisfying $0 \leq \lambda_{1}+\lambda_{2}<1$, then $A$ has a unique fixed point $x^{*}$ in $E$.

Proof. It follows from (6) that

$$
-\left(L_{1}+L_{2}\right)(x-y) \leq A(x)-A(y) \leq\left(L_{1}+L_{2}\right)(x-y), \quad x, y \in E, \quad x \geq y .
$$

Then all conditions of Theorem 3 are satisfied. 


\section{Applications to nonlinear differential equations}

Consider the nonlinear fractional boundary value problem given by

$$
\begin{gathered}
D_{0^{+}}^{\alpha} x(t)+f(t, x(t))=0 \quad 0<t<1, \\
x(0)=x^{\prime}(0)=x^{\prime}(1)=0 .
\end{gathered}
$$

where $f(t, x)$ is continuously differentiable on $0 \leq t \leq 1, x \in \mathbb{R}$ with derivative $f_{x}(t,$.$) and f(t, x) \geq 0$ for all $(t, x) \in[0,1] \times \mathbb{R}^{+} 2<\alpha<3$ is a real number and $D_{0^{+}}^{\alpha}$ is a standard Riemann-Liouville fractional derivative. Problems of the form (7) arise in many applications in physics, mechanics, chemistry and engineering, where usually the existence of non-negative solutions is of interest.

In this connection, we are interested in producing sufficient conditions for the existence of non-negative solution to (7).

For our considerations, we shall consider the Banach space $E=C[0,1]$ equipped with the standard norm

$$
\|x\|=\max _{0 \leq t \leq 1}|x(t)| .
$$

We define the normal cone $P$ by $P=\{x \in C[0,1]: x(t) \geq 0\}$.

Let $x \in C[0,1]$, then it is well-known that (see [12]) the boundary value problem (7) is equivalent to the following fractional integral equation:

$$
x(t)=\int_{0}^{1} G(t, s) f(s, x(s)) d s, \quad t \in[0,1]
$$

where

$$
G(t, s)= \begin{cases}\frac{(1-s)^{\alpha-2} t^{\alpha-1}}{\Gamma(\alpha)}, & 0 \leq t \leq s \leq 1 \\ \frac{(1-s)^{\alpha-2} t^{\alpha-1}}{\Gamma(\alpha)}-\frac{(t-s)^{\alpha-1}}{\Gamma(\alpha)}, & 0 \leq s \leq t \leq 1\end{cases}
$$

Here $\Gamma$ denotes the Gamma function.

The following lemma (see [12], Lemma 2.8) will be used later.

Lemma 10. $G(t, s) \geq t^{\alpha-1} G(1, s) \geq 0$ for $0 \leq s, t \leq 1$.

Now, consider the following operator

$$
F x(t)=\int_{0}^{1} G(t, s) f(s, x(s)) d s .
$$


Then equation (7) has a continuous, non-negative solution iff there exists $x \in P$ such that $x=F x$.

The following theorem gives sufficient condition so that (7) has a solution in $P$.

Theorem 11. Suppose that:

(F1) : there exists a continuous non-negative function $a:[0,1] \longrightarrow \mathbb{R}$ such that

$$
-a(t) \leq f_{x}(t, x) \leq a(t), \quad t \in[0,1], x \in \mathbb{R} .
$$

Then if

$$
a(t)<\frac{\Gamma(2 \alpha-1)}{\Gamma(\alpha-1)}, \text { for all } t \in[0,1],
$$

equation (7) has a unique non-negative solution $x \in C[0,1]$ and for any $x_{0} \in P$ the sequence defined by

$$
x_{n}(t)=\int_{0}^{1} G(t, s) f\left(s, x_{n-1}(s)\right) d s, \quad t \in[0,1], \quad n=1,2, \ldots
$$

converges to $x$ with $\left\|x_{n}-x\right\| \rightarrow 0$ as $n \rightarrow \infty$.

Proof. We are going to prove that all conditions of Theorem 8 are verified. For this, let $x, y \in E$ such that $x(t) \geq y(t), t \in[0,1]$. Then by the mean value theorem,

$$
f(t, x(t))-f(t, y(t))=f_{x}(t, \bar{x}(t))(x(t)-y(t))
$$

with

$$
y(t) \leq \bar{x}(t) \leq x(t)
$$

From which it follows that

$$
-L(x-y)(t) \leq F x(t)-F y(t) \leq L(x-y)(t),
$$

where $L: E \rightarrow E$ is the linear operator defined by

$$
L x(t)=\int_{0}^{1} G(t, s) a(s) x(s) d s, \quad t \in[0,1] .
$$

Let

$$
v_{0}(t)=\int_{0}^{1} G(t, s) d s=\frac{t^{\alpha-1}}{\Gamma(\alpha)}\left(\frac{1}{\alpha-1}-\frac{t}{\alpha}\right) .
$$


then for every non-zero element $x \in P$ we evidently have

$$
\int_{0}^{1} G(t, s) a(s) x(s) d s \leq\|x\|\|a\| v_{0}(t), \quad 0 \leq t \leq 1,
$$

on the other hand it follows from the inequality

$$
\frac{t^{\alpha-1}}{\Gamma(\alpha)}\left(\frac{1}{\alpha-1}-\frac{t}{\alpha}\right) \leq \frac{t^{\alpha-1}}{\Gamma(\alpha)}\left(\frac{1}{\alpha-1}\right)
$$

that the operator $L$ is $u_{0}$-bounded above where $u_{0}(t)=t^{\alpha-1}$.

A direct calculation shows that:

$$
\int_{0}^{1} G(t, s) s^{\alpha-1} d s=\int_{0}^{1} \frac{t^{\alpha-1}}{\Gamma(\alpha)}(1-s)^{\alpha-2} s^{\alpha-1} d s-\int_{0}^{t} \frac{(t-s)^{\alpha-1}}{\Gamma(\alpha)} s^{\alpha-1} d s .
$$

Then, by the substitution $s=\sigma t$ and the fundamental properties of the Beta functions we find that

$$
\begin{aligned}
\int_{0}^{1} G(t, s) s^{\alpha-1} d s= & =\frac{t^{\alpha-1}}{\Gamma(\alpha)} \frac{\Gamma(\alpha-1) \Gamma(\alpha)}{\Gamma(2 \alpha-1)}-\int_{0}^{1} t^{2 \alpha-1} \frac{(1-s)^{\alpha-1} s^{\alpha-1}}{\Gamma(\alpha)} d s \\
& =t^{\alpha-1} \frac{\Gamma(\alpha-1)}{\Gamma(2 \alpha-1)}\left(1-t^{\alpha} \frac{\alpha}{2 \alpha-1}\right) \\
& \leq t^{\alpha-1} \frac{\Gamma(\alpha-1)}{\Gamma(2 \alpha-1)} .
\end{aligned}
$$

From this, by virtue of (8) and since the function $a$ is continuous on $[0,1]$ we obtain that the operator $L$ satisfies the condition

$$
L u_{0} \leq \lambda^{\prime} u_{0}
$$

where $\lambda^{\prime}<1$.

Now, the conclusion can be deduced from Theorem 8. This completes the proof.

Remark 12. We must observe that if we take $\alpha(\alpha-1) \Gamma(\alpha)<a(t) \equiv a<$ $\frac{\Gamma(2 \alpha-1)}{\Gamma(\alpha-1)}$ then it can be shown that

$$
\|L\|=a \max _{t \in[0,1]} \int_{0}^{1} G(t, s) d s=\frac{a}{\alpha(\alpha-1) \Gamma(\alpha)}>1 .
$$


Here we use the fact that see $([6])$

$$
B(\alpha, \alpha-1)-\frac{1}{\alpha(\alpha-1)}>0, \quad \alpha>1, \quad \alpha-1>1
$$

where $B(x, y)$ denotes the Beta function $B(x, y)=\frac{\Gamma(x) \Gamma(y)}{\Gamma(x+y)}, \quad x, y>0$.

Remark 13. Note also that $u_{0}(t)=t^{\alpha-1} \notin \stackrel{\circ}{P}$.

\section{References}

[1] H. Amann, Fixed point equations and nonlinear eigenvalue problems in ordered Banach spaces, SIAM Rev., 18 (1976), 620-709.

[2] A. Cañada, and A. Zertiti, Topological methods in the study of positive solutions for some nonlinear delay integral equations, Nonlinear Analysis, T.M.A., 23, No 9 (1994), 1153-1165.

[3] A. Cañada and A. Zertiti, Fixed point theorems for systems of equations in ordered Banach spaces with applications to differential and integral equations, Nonlinear Analysis, T.M.A., 27 (1996), 397-411.

[4] D. Guo and V. Lakshmikantham, Nonlinear Problems in Abstract Cones, Academic Press, New York (1988).

[5] D. Guo, Y. Cho, and Z. Jiang, Partial Ordering Methods in Nonlinear Problems, Nova Science Publishers, New York (2004).

[6] H. Alzer, Sharp inequalities for the Beta function. Indagationes Mathematicae, 12, No 1 (2001), 15-21.

[7] M.S. El Khannoussi and A. Zertiti, Fixed point theorems in the study of operators equations in ordered Banach spaces, International Journal of Pure and Applied Mathematics, 4 (2016), 789-801.

[8] M.S. El Khannoussi and A. Zertiti, Topological methods in the study of positive solutions for operator equations in ordered Banach spaces. Electronic Journal of Differential Equations, No 171 (2016), 1-13.

[9] M.A. Krasnosel'skii, P.P. Zabreiko, Geometrical Methods of Nonlinear Analysis, Springer-Verlag, Berlin (1984). 
[10] M.A. Krasnosel'skii, Positive Solutions of Operator Equations, Noordhoff, Groningen (1964).

[11] M.G. Krein and M. Rutman, Linear operators leaving invariant a cone in a Banach space, Amer. Math. Soc. Transl., 10 (1962), 1-128 (Transl. from Uspekhi Mat. Nauk, 3 (1948), 3-95).

[12] Moustafa El-Shahed, Positive solutions for boundary value problems of nonlinear fractional differential equation, Abstract and Applied Analysis 2007 (2007), Art. 10368, 8 pages.

[13] J. Weissinger, Zur Theorie und Anwendung des Iterationsverfahrens. Math. Nachr., 8 (1952), 193-212.

[14] P.P. Zabreiko, M.A. Krasnosel'skii, and V.Ya. Stetsenko, Bounds for the spectral radius of positive operators. Translated from Matematicheskie $Z a$ metki, 1, No 4 (1967), 461-468. 